Acta Poetica $33 \cdot 2$

JULIO-DICIEMBRE

$2012(47-60)$

\title{
Dos chacales indios en la España medieval: notas para un estudio de la influencia de las fábulas indias en el nacimiento de la cuentística española
}

\author{
Wendy J. Phillips Rodríguez
}

De entrada pudiera parecer sorprendente que el primer ejemplo de ficción en prosa castellana del cual tenemos noticia, el Calila e Dimna, sea una traducción/adaptación de una obra clásica de la literatura sánscrita: el Pañcatantra. Sin embargo, amplios estudios textuales han logrado documentar las fases principales de la transmisión de dicha colección de cuentos desde la India antigua hasta la España medieval. Una veta de investigación que, en cambio, no se ha explorado es aquella que corresponde al análisis comparativo de las transformaciones que la obra sufrió en su largo peregrinaje para adaptarse a distintos contextos culturales. Este artículo es un paso hacia tal clase de estudios, ya que analizará algunas características de los personajes principales de la narración española, Calila y Dimna, e identificará sus similitudes y diferencias con sus ancestros indios: los chacales Karațaka y Damanaka.

PAlABRAS ClAVE: exempla, fábulas, literatura sánscrita, literatura medieval, transmisión textual.

At first it may seem unlikely to affirm that the first example of fictional Castilian prose available to us, Calila e Dimna, may be a translation/adaptation of the Pañcatantra, a classical work of Sanskrit literature. Nevertheless, the pilgrimage of such collection of tales from ancient India to Medieval Spain has been dutifully documented. On the other hand, a line of research that has not been explored yet is related to the comparative analysis of the transformations that such tales endured during their journey in order to adapt to new cultural environments. This paper is a first attempt towards such kind of studies. It will analyze some features of the two main characters of the Spanish version, Calila 
and Dimna, and will identify some similarities and differences in relation to their Indian ancestors: the jackals Karațaka and Damanaka.

Keywords: exempla, fables, Sanskrit Literature, Medieval Literature, textual transmission.

Fecha de recepción: 20 de febrero de 2012

Fecha de aceptación: 17 de abril de 2012 
Wendy J. Phillips Rodríguez

Instituto de Investigaciones Filológicas

Universidad Nacional Autónoma de México

\section{Dos chacales indios en la España medieval: notas para un estudio de la influencia de las fábulas indias en el nacimiento de la cuentística española}

Sabed que los de India pusieran proverbios et enxenplos.

Manuscrito P del Calila e Dimna, folio 137 v.

Hay historias que tienen una historia ellas mismas, a veces incluso tan fabulosa como los mismos eventos que nos narran. Tal es el caso de $\mathrm{Ca}$ lila e Dimna, el primer ejemplo de ficción en prosa en lengua castellana del cual tenemos noticia.

En los años cercanos al 1250 el infante Alfonso estaba a poco de convertirse en Alfonso X "el sabio", rey de la Corona de Castilla. En aquel tiempo la Península ibérica estaba llena de diferentes influencias culturales: judíos, árabes y cristianos populaban el territorio español impregnándolo con sus diferentes culturas y religiones. Las lenguas en uso eran el árabe, el hebreo, el latín de la Iglesia y las distintas formas de latín romanzado que se hablaban (pero no se escribían) en las diferentes provincias de España. Ante un escenario tan multicultural Alfonso tomó, todavía siendo infante, la decisión que lo haría famoso como rey: rodearse de un grupo de hombres sabios de las distintas tradiciones y comenzar un taller de traducción en la ciudad de Toledo. En ese taller textos del latín, del hebreo y del árabe fueron traducidos, por escrito, a 
la forma vernácula del latín que se usaba en la provincia de Castilla: el castellano ("el nuestro lenguaje de Castiella", según palabras del mismo Alfonso). Así fue como nació lo que hoy conocemos como lengua española. Así fue, también, como una decisión linguística se convirtió en la más importante determinación política tomada por el soberano, determinación cuyas consecuencias nos alcanzan hasta la actualidad:

Las decisiones sobre las lenguas son consideradas una cuestión política que incide en el desarrollo de naciones y regiones, la construcción de identidades colectivas, y el intercambio comercial, científico y cultural de los pueblos (Barroso, "Alfonso X”, 2).

De todos los textos que pudieron haber sido traducidos en el taller de Toledo, el primero del cual tenemos noticia es el Calila e Dimna, una colección de historias cuyo núcleo proviene de una obra clásica de la literatura sánscrita: el Pañcatantra. ${ }^{1}$ El Pañcatantra, cuya traducción literal es cinco (pañca) libros o principios (tantra), es un texto muy difundido pero de cuyo origen se sabe poco. Se cree que la colección completa de historias, tal y como ahora la conocemos, fue recopilada alrededor del siglo III a. C. Sin embargo, es muy probable que varias de esas historias existieran previamente y fueran parte de la tradición oral sánscrita. Sobre el Pañcatantra Franklin Edgerton nos dice:

Es, ciertamente, el producto literario de la India que ha sido más frecuentemente traducido, y sus historias se encuentran entre las más conocidas de todo el mundo [...] Se tiene registro de más de doscientas versiones en más de cincuenta lenguas, tres cuartas partes de estas lenguas no son indias. Para el siglo xI este texto ya había llegado a Europa y aun antes del 1600 ya existían (versiones) en griego, latín, español, italiano, alemán, inglés, eslavo antiguo, checo y quizá en otras lenguas eslávicas. Su alcance se ha extendido desde Java hasta Islandia [...] en India ha sido retomado una y otra vez, se ha expandido, reducido, puesto en verso, vuelto a contar en prosa, traducido a lenguas medievales y vernáculas y luego vuelto a retraducir al sánscrito (The Panchatantra, 3).

${ }^{1}$ La relación entre el Calila e Dimna y el Pañcatantra ha sido ampliamente documentada. Véase Benfey, Pantschatantra. 
Es así como en la historia de la literatura española el primer ejemplo de ficción en prosa es un texto que originalmente nació en el seno de la cultura clásica de la India, y el cual, precisamente por ser el primero, marcó también el desarrollo y la tesitura que adquiriría la cuentística española en sus primeras etapas, como lo afirma Gómez Redondo:

El Calila es la primera colección de cuentos orientales traducida al castellano, en un momento clave para la formación del pensamiento literario peninsular y para la construcción del discurso prosístico, justo en la mitad del siglo XIII, cuando Alfonso — bien como infante, bien como rey- está dotando de sentido a ese "lenguaje de Castiella" al que quiere convertir en adecuado medio de expresión del "saber" para organizar el espacio político e ideológico de su corte (Historia de la prosa, 182).

Sin embargo, hay que tener en cuenta que en aquel tiempo traducción quería decir, en gran medida, adaptación. La historia de los dos chacales tuvo un largo viaje lleno de aventuras desde la India antigua hasta la España del siglo XIII. Para adaptarse a sus cambiantes contextos lingüísticos, políticos, religiosos y culturales tuvo que sufrir una larga serie de modificaciones, de tal suerte que de la India a España hubo un gran número de versiones intermedias. La crítica textual occidental se ha ocupado de trazar la historia de tales versiones, esperando construir un mapa coherente de la ruta seguida por estos cuentos en su peregrinaje de Asia a Europa. ${ }^{2}$

Sabemos que la primer versión no india fue una traducción al pehlevi (persa medio) alrededor del 570 d. C. por un sabio llamado Berzebuey, la cual a su vez fue traducida al siriaco y al árabe alrededor del siglo XIII. La versión al árabe, atribuida a Ibn al-Muqaffa', fue la que jugó un rol importantísimo en la diseminación de las historias en diferentes culturas del mundo. Hasta ahora se han identificado tres grupos de traducciones que surgieron a partir de esta versión árabe:

a. Una nueva versión persa (siglo XII), que dio lugar a posteriores versiones en persa (siglo XVI) y en turco (siglo XVII).

${ }^{2}$ Para una representación gráfica de la historia de la transmisión del texto véanse las introducciones de Cacho Blecua y Lacarra, Calila e Dimna, 64 y Villegas, Calila y Dimna, 21. 
b. Dos traducciones al hebreo (siglo XIII), una de las cuales fue después traducida al latín por J. de Capua (finales del siglo XIII).

c. La versión "Alfonsí" en castellano ( siglo XIII), de la cual se conservan tres manuscritos, dos completos (A y B, en la biblioteca del Real Monasterio de San Lorenzo del Escorial), y un fragmento (P, en la biblioteca universitaria de Salamanca). ${ }^{3}$

A lo largo de su viaje la obra se modificó de formas distintas. De India a España hubo varias versiones intermedias, y cada una de ellas representó una fase evolutiva por medio de la cual la historia fue despojándose poco a poco de los detalles específicos referentes a la cultura india con el propósito de convertirse en una obra de interés universal. Como resultado, la versión castellana, a pesar de provenir claramente del Pañcatantra contiene también un gran número de innovaciones y diferencias.

El texto sufrió de pérdida, adquisición y reestructuración del material original. Muchos cuentos, incluso libros enteros del Pañcatantra se perdieron en el camino, mientras que otras historias, provenientes de diferentes colecciones, fueron insertadas. Además, relatos pertenecientes al folclor de las culturas intermedias se hicieron camino dentro del texto gracias a la receptividad de su estructura de "cuentos de cajón". Tal estructura consiste en contar un cuento dentro de otro cuento que le sirve de marco (generalmente un personaje de la historia principal decide narrar algo, dando pie así para la intromisión de un nuevo relato dentro de la narración existente sin que ello afecte directamente la continuidad de la historia marco). La técnica narrativa de los cuentos de cajón, que puede alcanzar altos grados de complejidad al manejar varios niveles narrativos, permite una gran movilidad de material sin perjuicio de la estructura de la obra, cuya esencia es precisamente el acoger o desechar historias de acuerdo con el propósito que se tenga en el momento, ya que como afirman Cacho Blecua y Lacarra en el estudio introductorio a su edición: "el número de relatos insertados

3 Menéndez Peláez (“Un fragmento desconocido", 167-172) descubrió otro fragmento muy breve, solamente 22 líneas, de la introducción de Ibn al-Muqaffa'. 
puede variar y modificarse el argumento de ellos, no solo en cada versión, sino a veces en cada manuscrito" ("Introducción", 11).

Por tal razón lo más notable no es solo la aparición o desaparición de algunas de las historias internas, sino el hecho de que a la historia general se le haya agregado un nuevo marco narrativo, o mejor dicho, dos. El marco principal del Pañcatantra nos habla sobre los esfuerzos del sabio Viṣnuśarman por educar a los hijos malcriados del rey Amaraśakti, lo cual logra por medio de historias didácticas que terminan siempre con una moraleja. Los cinco capítulos que conforman el libro son cinco lecciones distintas sobre los temas importantes que los príncipes deben aprender: cómo las amistades pueden romperse por medio de la intriga, cómo conseguir amigos, cómo hacer la guerra, cómo las ganancias adquiridas pueden perderse si no se cuidan y cómo no actuar de manera precipitada. En cambio, la historia marco del $\mathrm{Ca}$ lila e Dimna inicia con el discurso del mismo Ibn al-Muqaffa'-quien así deja de ser un personaje propiamente histórico y se convierte en uno de ficción- en el cual nos informa sus razones para tratar de "trasladar del lenguaje de Persia al lenguaje arábigo" (98) la obra que nos ocupa. Además, Ibn al-Muqaffa' agrega que "et quesimos et tovimos por bien de atraer en él un capítulo de arávigo en que se mostrase el escolar diçípulo en la fazienda deste libro...", con lo cual introduce el segundo marco narrativo que nos cuenta sobre el viaje del sabio persa Berzebuey a la India, enviado por el rey Sirechuel, en busca de unas hierbas medicinales que se encontraban en lo alto de una montaña y que tenían la capacidad de resucitar a los muertos. Esta historia relata cómo después de visitar varios reinos Berzebuey es incapaz de encontrar tales hierbas y se siente avergonzado de regresar a Persia con las manos vacías. Al percibir su angustia los sabios indios le revelan el significado simbólico de la medicina que está buscando: aquellas hierbas representan la sabiduría, la cual es capaz de resucitar a los "omnes nesçios que non saben quándo son melezinados en el saber" (101), quienes al vivir en la ignorancia es como si estuvieran muertos porque desconocen el verdadero significado de la vida. Berzebuey, entonces, se aboca a "despertar" al hombre a la verdadera vida (la vida en el conocimiento) y con la ayuda de los sabios indios logra traducir los textos a la lengua persa y regresar a casa para presentárselos a su rey. Y resulta, 
precisamente, que "uno de aquestos escriptos es aqueste libro de Calila et Dina" (102).

De esa forma la obra se presenta a sí misma como un texto lleno de sabiduría extraída con gran esfuerzo de tiempos y espacios remotos. Con sus nuevos marcos narrativos el Calila logra envolver las prácticas enseñanzas de nīti (la ciencia política, la ciencia de la conducta) ${ }^{4}$ en un aura de misterio, y consigue que la obra se presente a sí misma con todas las propiedades de un conocimiento salvífico y lleno de simbolismos. La aventura de Berzebuey en busca de la hierba medicinal (marco 2), relatada después de la "Introducción" de Ibn al-Muqaffa' (marco 1), termina así adquiriendo proporciones iniciáticas: la montaña representa el conocimiento, alzándose siempre por encima de la multitud; los muertos son los hombres que no tienen sabiduría; la hierba que él busca se encuentra en lo más alto de la montaña porque es tierna y fácil de digerir (como lo son las pequeñas historias relatadas), pero se requiere del mayor esfuerzo para llegar a ella (porque tales historias, en su simplicidad, no son sino la condensación de los grandes tratados del saber). ${ }^{5}$ Asimismo, al incorporar dentro de sí misma la historia de su transmisión, el Calila adquiere una suerte de circularidad que la convierte en una historia que cuenta su propia historia.

Siguiendo con el esquema narrativo del Calila, después de la "Introducción" de Ibn al-Muqaffa' y enseguida de la descripción de cómo Berzebuey llevó a cabo su misión (Capítulo I, "Cómmo el rey Sirechuel enbió a Berzebuey a tierra de India") aparece una sección en la que él mismo cuenta la historia de su vida (Capítulo II, "Estoria de Berzebuey el Menge") y es solo hasta el Capítulo III donde propiamente entra-

${ }^{4}$ El Pañcatantra pertenece a un grupo de textos conocidos como nītiśāstra, que se caracterizan por impartir enseñanzas relacionadas con la sabiduría mundana. A menudo sus lecciones se preocupan más por obtener el objetivo deseado que por mantener una determinada postura moral.

${ }^{5}$ Hay que recordar que el valor de este tipo de historias es hacer asequible un enorme caudal de conocimiento que de otra forma tomaría años (y grandes esfuerzos) aprender. En el Pañcatantra el rey Amaraśakti decide confiar la educación de sus hijos a Viṣnuśarman porque es el único que le asegura que, con su método, los príncipes habrán aprendido todo en seis meses: putrānmāsașațena yadi nītiśāstrajñānna karomi tatah svanāmatyāgam karomi ("si no logro que sus hijos aprendan nītiśāstra —sabiduría mundana-enseismesesrenunciaréhasta ami propionombre”, Kale, ed.,Pañcatantra,3). 
mos en materia y encontramos la historia "Del león et del buey" que es precisamente la fábula con la que inicia el Pañcatantra y en la que por primera vez aparecen Karațaka y Damanaka (por sus nombres en sánscrito), dos chacales que procuran ganarse el favor del rey león por medio de intrigas, y a los cuales encontraremos en la traducción alfonsí, hispanizados de varias maneras, pero siempre distinguiéndose por ser "muy ardides et agudos". ${ }^{6}$ El resto del libro, la parte que propiamente proviene del Pañcatantra y que consiste de fábulas más o menos sueltas, se articula por medio del diálogo ficticio entre un rey y un filósofo —de personalidades muy difusas - en el que a petición del monarca el filósofo cuenta una serie de historias ejemplares con el propósito de ilustrar sobre diferentes aspectos del saber.

Como ya se había mencionado, el Pañcatantra fue remodelado y hubo un reacomodo de su material narrativo. Para empezar, la que era una historia más dentro de otra historia se convirtió en la historia marco. Como consecuencia, Karațaka y Damanaka, los dos chacales astutos que solo eran importantes en los dos primeros capítulos del Pañcatantra se convirtieron así en los personajes principales de la obra. Aparentemente este cambio tuvo lugar desde la primera versión árabe en el siglo VIII. También, el nombre de los dos chacales sufrió transformaciones para adecuarse a la pronunciación y a los hábitos lingüísticos de las diferentes culturas. En su camino a España, Karațaka se convirtió en Calila y Damanaka se convirtió en Dimna. ${ }^{7}$ Pero no solamente su estatus narrativo y sus nombres cambiaron, sino que su propia natura-

${ }^{6}$ Cabe aclarar que aunque los dos personajes son astutos, hay una diferencia de personalidad entre ellos que aparecía ya en el Pañcatantra y que en el Calila se presenta de la siguienta forma: "Et era Dina de más noble coraçón, et de mayor fazienda, et el que menos se tenía pagado del estado en que era".

${ }^{7}$ En sánscrito, los nombres de los chacales tienen un significado adecuado a sus personalidades: karata quiere decir hombre ruin, un mal brahmán, un ateo o descreído, alguien que no sigue los preceptos sagrados. Damana, en cambio, es un adjetivo que hace referencia a alguien que tiene control sobre sí mismo. El sufijo $k a$, presente en ambos nombres, es utilizado comúnmente para formar diminutivos, deprecativos o para expresar similitud y su significado esencial es "que tiene las características de". Una vez que esta historia es traducida a otras lenguas los nombres siguen guardando una cierta semejanza fonética con sus originales, sin embargo - por razones obvias - sus significados se pierden. 
leza de chacales tuvo que ser modificada para adecuarse a sus nuevos contextos:

Los que nacieron chacales en la India, es decir, "mamíferos carnívoros de la familia de los cánicos", pasaron a ser linces en España, "mamíferos carniceros" muy parecidos a un tipo de gato llamado montés. Esta metamorfosis se dio por una razón muy sencilla: en el siglo xIII, cuando se hizo la traducción del árabe al castellano, en España se desconocía totalmente la especie del chacal. La palabra "chacal" no ingresó a nuestro idioma sino hasta medio milenio más tarde, entre 1765 y 1783, y eso por vía europea, no asiática ni africana. Sin embrago, hay que admitir con toda honradez que el término "lince", procedente del latín lynx, lyncis, y este del griego l'ynx, lynkós, tampoco existía en España en el momento de la traducción del árabe al castellano de Kaliila wa-Damna (recibió carta de naturalización en 1490). Los dos personajes en discusión, vasallos del rey león y miembros de su corte, eran "lobos cervales" -manera antigua de describir al lince, animal cuya personalidad encajaba perfectamente con el carácter y las acciones de Calila y Dymna (Jarocka, El coloquio, 14).

Es precisamente en este tipo de datos, aparentemente de importancia solamente anecdótica, donde se pueden observar con mayor claridad los mecanismos de actualización que ocurren en las narrativas para hacerse relevantes para nuevas audiencias. Aunque la fábula de los dos chacales, como historia, era fácilmente adaptable a un nueva cultura, la identidad de los chacales debía transformase para así poder generar interés entre sus nuevos receptores, porque ¿para qué contar una historia sobre chacales a un público que jamás ha visto uno? En tal caso es más efectivo utilizar a un animal que sea ampliamente conocido y que posea las mismas cualidades, en este caso suspicacia y sagacidad, para que así el público logre entender fácilmente el desarrollo de la historia. ${ }^{8}$ Cabe mencionar, entonces, que la especie animal a la cual corresponde el nombre común de lobo cerval (lynx pardinus) es un tipo de felino endémico de la península ibérica y diferente del lince euroasiático (lynx

${ }^{8}$ Hay que recordar que el uso de animales para estereotipar carácteres humanos es uno de los recursos más efectivos de la fábula, ya que así se logra contar una historia en forma muy condensada, sin detenerse en descripciones psicológicas de los personajes o en sus motivaciones. 
lynx) que suele encontrarse en otras partes de Europa. Aunque actualmente el lobo cerval es una de las pocas especies felinas en peligro de extinción, en el tiempo de la traducción alfonsí debió haber sido un mamífero conocido por su agilidad para la caza (siendo la liebre su presa favorita), lo cual para efectos de la narración sería una característica equivalente a la agilidad mental.

Hay que mencionar, sin embargo, que en las traducciones medievales de cuentos orientales el chacal indio no siempre fue directamente transformado en lobo cerval. Por ejemplo, sabemos que "R. Llull, cuando hacia 1285-1286 redactó su Llibre de les bèsties, conocía total o parcialmente el Calila. En el marco narrativo se identifican claramente el buey, el león y el personaje de Dimna, ahora encarnado en zorro" (Cacho Blecua y Lacarra, "Introducción", 13). Sin embargo, también sabemos que la adaptación de Llull, por sus severas discrepancias con la versión alfonsí del Calila, estuvo basada en una versión diferente (quizá oral, quizá escrita) de la que fue traducida por la escuela de Toledo. Asimismo, en una nota a pie de página, Cacho Blecua y Lacarra ofrecen la siguiente información:

El chacal, en opinión de Th. Benfey, equivale en los textos hindúes al zorro de las fábulas occidentales y se caracteriza por su astucia. Suele aparecer asociado al león, ya que, según creencia popular, el chacal sigue las huellas del león para comer lo que este abandona (25).

Probablemente esto se deba a la misma naturaleza del chacal que, como lo describe el Diccionario de la RAE en su $22^{\mathrm{a}}$ edición, es un "mamífero carnívoro de la familia de los Cánidos, de un tamaño medio entre el lobo y la zorra, parecido al primero en la forma y el color, y a la segunda en la disposición de la cola". Claramente, distintos autores eligieron diferentes animales para tomar la personalidad del chacal, pero en todos los casos optaron por animales conocidos en el medio local para substituirlo. ${ }^{9}$

${ }^{9}$ Quizá sea de interés mencionar que el segundo manuscrito más antiguo que se conserva del Kalila wa Damna —versión árabe del texto en cuestión-, con fecha 747 Héjira (año 1346 de nuestra era aprox.) y actualmente preservado en el King Faisal Center for Research and Islamic Studies, contiene varias ilustraciones en miniatura en 
Como hemos visto fue solo después de una larga serie de transformaciones que Karațaka y Damanaka estuvieron listos para convertirse en los emblemáticos lobos cervales que la literatura española siempre ha considerado como los dos primeros personajes de su ficción en prosa.

Otro aspecto relevante de la historia de la transmisión de este texto es que solo se empezó a explorar a partir del siglo XIX, cuando las afinidades entre el sánscrito y otras lenguas indoeuropeas comenzaron a ser identificadas. Durante largos siglos se había olvidado cuál era la fuente original del Calila e Dimna. Lo único que se sabía es que provenía de cuentos del lejano oriente pero no se había identíficado su fuente específica, y por lo tanto tampoco se habían estudiado las trasformaciones que la historia había sufrido en su peregrinaje.

Quizá como resultado de ese olvido, la literatura española abrazó los cuentos como si fueran propios. El elemento de misterio: "provienen de oriente pero no se sabe exactamente de dónde", logró hacerlos más atractivos para las audiencias que probablemente veían en ellos un aura de magia y exotismo. Lo más notable es que la literatura española tomó no solamente un número finito de historias como el Calila e Dimna, sino que aprendió rápidamente el arte de contar cuentos "a la manera de" la India antigua. Una vez que la joven literatura española asimiló la técnica de insertar una narración dentro de otra y probó la eficacia de educar por medio de historias moralizantes se convirtió en una portentosa fábrica de cuentos. ${ }^{10}$ De tal suerte que el dicho popular de que "todos los cuentos vienen de la India" alude más al hecho de haber aprendido una manera de narrar, que a un número determinado de historias. A razón de ello la cuentística medieval española acusa un cierto sabor indio (e.g. El Conde Lucanor, El caballero Zifar, etc.) aun cuando no todas las historias que cuenta provengan necesariamente del subcontinente. En otras palabras, más que haber importado el producto hecho, lo que se tomó de la tradición fabulística india fue el know-how de cómo producirlo en casa, utilizando los materiales a la mano. Pronto, la recién nacida literatura española comenzó a tomar inspiración de

las que Calila y Dimna aparecen como chacales. Lo anterior da cuenta de que en los lugares donde el chacal era un animal común la naturaleza de los personajes no sufrió ninguna modificación.

${ }^{10}$ Véase Marcelino Villegas, “Introducción”, 25. 
fuentes bíblicas, clásicas y populares y las utilizó de forma libre para crear nuevas colecciones de historias. El género llamado "exempla" (que contiene siempre una narración de tipo didáctico), tan común en la España medieval, incorporó muchas características del Calila e Dimna y de otros trabajos similares que fueron traducciones / adaptaciones de obras originarias de la India. ${ }^{11}$ Es por ello que podemos afirmar que las fábulas indias, su estructura y su afán didáctico, tuvieron un papel seminal en el desarrollo de la cuentística española.

Es claro que no solo España, sino también otros países europeos vieron influenciada su literatura por las fábulas indias. Sin embargo, el caso de España es particular porque la dominación árabe del territorio español (del 711 al 1492) coincide, en gran parte, con la presencia de los pueblos musulmanes en territorio indio. Fue precisamente gracias al mundo árabe, y a su mediación como puente comunicador, que dos culturas tan lejanas geográficamente lograron estar en contacto. Es por ello que las conexiones culturales entre España y la India no son tan improbables como pudieran parecerlo por principio. A medida que progresen los estudios indológicos en el mundo hispano será posible estudiar más a fondo hasta qué punto la cuentística española, en sus primeras etapas, abrevó en el río sonoro de la literatura sánscrita.

\section{REFERENCIAS}

Benalmocaffa, Abdalá, Calila y Dimna, Marcelino Villegas (trad.), Madrid, Alianza, 1991.

BArroso, Graciela, "Alfonso X y la escuela de traductores de Toledo: notas para un estudio de políticas sociolingüísticas", Actas Academia Luventicus 5, 2003, 1-10.

Benfey, Theodor, Pantschatantra. Fünf Bücher indischer Fabeln, Märchen und Erzählungen. Aus dem Sanskrit Übersetzt mit Einleitung und Anmerkungen versehen, Leipzig, Brockhaus, 1859.

Cacho Blecua, Juan Manuel y María Jesús Lacarra, "Introducción” a Calila e Dimna, Madrid, Clásicos Castalia, 1984, 9-71.

${ }^{11}$ Otros dos ejemplos bien documentados de colecciones de historias de proveniencia india en la literatura española son El libro del los engaños o Sendebar y la historia de Barlaam y Josafat. 
Calila e Dimna, Juan Manuel Cacho Blecua y María Jesús Lacarra (eds.), Madrid, Clásicos Castalia, 1984.

EdgerTon, Franklin, The Panchatantra Reconstructed (vol. 1: Text and Critical Apparatus, vol. 2: Introduction and Translation), Connecticut, volumes 2-3 of American Oriental Series, 1924.

Gómez Redondo, Fernando, Historia de la prosa castellana, vol.1, Madrid, Cátedra, 1998.

JarockA, Marja Ludwicka, El coloquio de los perros a una nueva luz, México, Universidad Nacional Autónoma de México, 1979.

Kale, M. R. (ed.), Pañcatantra of Vishnusharman, Delhi, Motilal Banarsidass, 2008 (reimpresión).

Menéndez Peláez, Jesús, "Un fragmento desconocido del Calila e Digna en un manuscrito de la catedral de Oviedo", Revista de Literatura, 1983, 167172.

VilLEGAS, Marcelino, "Introducción" a Calila y Dimna (traducción del árabe de la versión de Abdalá Benalmocaffa), Madrid, Alianza, 1991. 